647

\section{COMPARISON OF BALANCED 6\% HES SOLUTION TO UNBALANCED $6 \%$ HES SOLUTION IN HYPOVOLEMIC CRITICAL ILL CHILDREN}

\author{
V. Van Gorp, S. Hachimi-Idrissi \\ Pediatric Critical Care Department (VUB), \\ UZ Brussel, Brussels, Belgium
}

Goal of the study: The third generation of unbalanced hydrxyethyl starch (HES) (130/0.4) (Voluven $®)$ shows favorable physicochemical properties and avoids negative effects on coagulation. Recently, increasing evidence reported that balanced, plasma-adapted HES preparation (Volulyte ${ }^{\circledR}$ ) has advantage over the unbalanced HES solution in chirurgical adult population and avoids hyperchloremic acidosis. We compare the efficacy Volulyte to Voluven in non chirurgical hypovolemic critically ill children.

Materials and Methods: Up to $40 \mathrm{ml} / \mathrm{kg}$ of the study fluid was infused until stabilization in 20 pediatric patients admitted with circulatory or respiratory failure with shock. The hemodynamic parameters, volume of the study fluid, biological analysis as well as the evolution of the $\mathrm{pH}$, base excess, bicarbonate, chloride and anion gap were analyzed before, after and 24 hours later after administration.

Results: After fluid administration and hemodynamic stabilization, no difference in respiratory frequency, ventilatory conditions, kidney, hematological or coagulation values between different treatment groups was observed.In Voluven group $(n=10$ patients) however, there was a significantly higher need for treatment volume per kg body weight. The $\mathrm{pH}$, serum base excess, and anion gap were significantly higher in the Volulyte group ( $n=$ 10 patients) while the serum chloride remained significantly lower when compared to the Voluven group whereas the serum bicarbonate remained stable.

Conclusion: The two study fluid showed no difference in term of hemodynamic, coagulation or kidney functions. The Volulyte group showed however, a clear lower serum chloride values as well as less acidosis as indicated by a higher $\mathrm{pH}$ and base excess.
648

\section{OESOPHAGEAL DOPPLER MONITOR DETECTS CHANGES IN CARDIAC INDEX DURING PACEMAKER TESTING IN CHILDREN AFTER CARDIAC SURGERY}

T. Fleck ${ }^{1,2}$, S. Schubert ${ }^{2}$, B. Stiller ${ }^{1}$, M. Redlin ${ }^{3}$, P. Ewert ${ }^{2}$, N. Nagdyman², F. Berger ${ }^{2}$

${ }^{1}$ Congenital Heart Disease/ Pediatric Cardiology, Centre for Pediatrics/ University Medical Centre

Freiburg, Freiburg, ${ }^{2}$ Department of Congenital Heart Disease/ Pediatric Cardiology, ${ }^{3}$ Department of Anesthesiology, Deutsches Herzzentrum Berlin, Berlin, Germany

Background: The Cardio QP ${ }^{\mathrm{TM}}$ Oesophageal Doppler Monitor measures the velocity time integral of the blood flow in the descending aorta. Based on nomogramms of the aortic cross sectional area of a paediatric population cardiac index is calculated and displayed on the monitor.

Objective: Evaluation of the capability of the Oesophageal Doppler Monitor to detect slight changes in cardiac output as they occur during desynchronizing ventricular pacing (VVI) in children after cardiac surgery.

Patients: 11 infants (6 female, 5 male) after corrective cardiac surgery of congenital heart defects. Median age: 4.9 months (2.0-15 months), median weight: $4.4 \mathrm{~kg}(3.4-7.8 \mathrm{~kg})$.

Interventions: After baseline measurements of cardiac output, we performed 3 steps of each 5 minutes: 1. ventricular- pacing, 2. baseline II , 3 . atrial pacing in order to exclude effects of higher cardiac frequency. We measured the effects on hemodynamic and respiratory parameters as well as on cardiac output.

Measurements and Main Results: VVI pacing leads to a significant decrease in arterial blood pressure and central venous saturation. Cardiac output parameters measured by the oesophageal Doppler monitor showed a significant decrease in stroke volume (SV) from $4.9 \pm 2.2$ to $4.2 \pm 2.1(p=$ $0.005)$ and cardiac Index $(\mathrm{Cl})(2.6 \pm 1.1$ to $2.1 \pm$ $0.8)(p=0.009)$ from baseline during ventricular stimulation. The changes in Cardiac output and hemodynamic parameters during atrial stimulation did not show a significant difference from baseline.

Conclusion: The oesophageal Doppler monitor may be reliable in the detection of slight changes in cardiac output. 